\title{
Conformal Field Theory Correlators from Classical Field Theory on Anti-de Sitter Space II. Vector and Spinor Fields
}

\author{
W. Mück* and K. S. Viswanathan ${ }^{\dagger}$ \\ Department of Physics, Simon Fraser University, Burnaby, B.C., V5A 1S6 Canada
}

February 1, 2008

\begin{abstract}
We use the AdS/CFT correspondence to calculate CFT correlation functions of vector and spinor fields. The connection between the AdS and boundary fields is properly treated via a Dirichlet boundary value problem.
\end{abstract}

*e-mail: wmueck@sfu.ca

†e-mail: kviswana@sfu.ca 


\section{Introduction}

The study of conformal field theories (CFT's) in dimensions larger than 2 [1], 2] has recently been boosted by Maldacena's conjecture that the large $N$ limit of certain conformal field theories in $d$ dimensions can be described by supergravity and string theory on $d+1$ dimensional Anti-de Sitter (AdS) space [3]. Subsequently, this conjecture has been given a more precise formulation [4, 5] and it has been shown that, in fact, any field theory on $\mathrm{AdS}_{d+1}$ is linked to a conformal field theory on the AdS boundary [5]. This observation is entirely due to the fact that one obtains a metric on the AdS boundary by multiplying the AdS metric with a function, which has a single zero on the boundary in order to counteract the divergence of the AdS metric. However, this function is otherwise arbitrary, which imposes the symmetries of the conformal group on the boundary metric. All one needs then is a suitable connection between the fields on $\mathrm{AdS}_{d+1}$ and its boundary. Schematically, this connection is given by

$$
Z_{A d S}\left[\phi_{0}\right]=\int_{\phi_{0}} \mathcal{D} \phi \exp (-I[\phi]) \equiv Z_{C F T}\left[\phi_{0}\right]=\left\langle\exp \left(\int d^{d} x \mathcal{O} \phi_{0}\right)\right\rangle,
$$

where $\phi_{0}$ is a suitably defined boundary value of the AdS field $\phi$ and couples as a current to the boundary conformal field theory operator $\mathcal{O}$. In the classical approximation the path integral on the l.h.s. is, of course, redundant.

Field theories on AdS spaces have been the subject of research in the past [6, 7, 8, 9, 10, 11, 12, 13, 14. More recently, the AdS/CFT correspondence has been investigated for

scalar fields [15, 16, 17], gauge fields [17], spinors [18], classical gravity [19] and type IIB string theory [20, 21]. For a comprehensive list of recent references see [17].

Using as representation of $\operatorname{AdS}_{d+1}$ the upper half space $x_{0}>0, x_{i} \in \mathbb{R}$, with the metric

$$
d s^{2}=\frac{1}{x_{0}^{2}} d x^{\mu} d x^{\mu}
$$

$(\mu=0,1, \ldots d)$, its boundary is compactified $\mathbb{R}^{d}$ (the points with $x_{0}=0$ and the single point $\left.x_{0}=\infty\right)$. We will frequently denote AdS vectors by $\left(x_{0}, \mathbf{x}\right)$ and use $x_{i}$ to specify the components of $\mathbf{x}$.

The fact that the AdS metric diverges on the boundary presents a difficulty in the AdS/CFT correspondence, which is to be met with care. The natural solution is to calculate the AdS action on a surface $x_{0}=\epsilon$ and then take the limit $\epsilon \rightarrow 0$. However, the exact connection between the AdS fields $\phi$ and the boundary fields $\phi_{0}$ is subtle. Whereas Witten [5] stated that $\phi$ should approach $\phi_{0}$ times a certain power of $x_{0}$ as $x_{0} \rightarrow 0$, it was soon realized [17] that in certain cases, in order to satisfy Ward identities, one must formulate a proper Dirichlet boundary value problem on the surface $x_{0}=\epsilon$ and take the limit $\epsilon \rightarrow 0$ at the very end. A detailed investigation taking into account this subtlety has so far been 
done only for scalar fields [16, 17]. We find it therefore necessary to extend our previous investigation of the scalar field [16] to the vector and Dirac fields on $\mathrm{AdS}_{d+1}$. To be general, we shall include a mass term in the vector field action, which is considered in Sec. 2. In Sec. 3 we will give account of the Dirac field. The minimal coupling of the Dirac and gauge fields is considered in Sec. 田, and Sec. 0 contains conclusions.

\section{The Vector Field}

The starting point is the action

$$
I=\int d^{d+1} x \sqrt{g}\left(\frac{1}{4} F_{\mu \nu} F^{\mu \nu}+\frac{1}{2} m^{2} A_{\mu} A^{\mu}\right)
$$

with the usual relation $F_{\mu \nu}=\partial_{\mu} A_{\nu}-\partial_{\nu} A_{\mu}$. The equation of motion derived from (3) is

$$
\nabla_{\mu} F^{\mu \nu}-m^{2} A^{\nu}=0
$$

which implies the subsidiary condition

$$
\nabla_{\mu} A^{\mu}=0
$$

Within our representation of Anti-de Sitter space (2) one can use (4) and (5) to obtain an equation for $A_{0}$,

$$
\left[x_{0}^{2} \partial_{\mu} \partial_{\mu}+(1-d) x_{0} \partial_{0}-\left(m^{2}-d+1\right)\right] A_{0}=0 .
$$

Introducing $\tilde{m}^{2}=m^{2}-d+1$ we know from the consideration of the scalar field that the solution of (6), which does not diverge for $x_{0} \rightarrow \infty$, is given by

$$
A_{0}(x)=\int \frac{d^{d} k}{(2 \pi)^{d}} \mathrm{e}^{-i \mathbf{k} \cdot \mathbf{x}} x_{0}^{\frac{d}{2}} a_{0}(\mathbf{k}) K_{\tilde{\alpha}}\left(k x_{0}\right) .
$$

with

$$
\tilde{\alpha}=\sqrt{\frac{d^{2}}{4}+\tilde{m}^{2}}=\sqrt{\frac{(d-2)^{2}}{4}+m^{2}} .
$$

It is useful to introduce fields with Lorentz indices by

$$
\tilde{A}_{a}=e_{a}^{\mu} A_{\mu}=x_{0} A_{a}
$$

where $e_{a}^{\mu}$ denotes the vielbein $(a=0,1, \ldots d)$. The virtue of this is seen when considering the components $\tilde{A}_{i}(i=1,2, \ldots d)$, whose equation of motion is again obtained from (4) and (5) and is given by

$$
\left[x_{0}^{2} \partial_{\mu} \partial_{\mu}+(1-d) x_{0} \partial_{0}-\tilde{m}^{2}\right] \tilde{A}_{i}=2 x_{0} \partial_{i} \tilde{A}_{0}
$$


The solution of the homogeneous part of (10) can be taken over from $A_{0}$ and the inhomogeneous equation is solved by making a good guess as to which form the solution should have. One obtains

$$
\tilde{A}_{i}(x)=\int \frac{d^{d} k}{(2 \pi)^{d}} \mathrm{e}^{-i \mathbf{k} \cdot \mathbf{x}} x_{0}^{\frac{d}{2}}\left(a_{i}(\mathbf{k}) K_{\tilde{\alpha}}\left(k x_{0}\right)+i a_{0}(\mathbf{k}) \frac{k_{i}}{k} x_{0} K_{\tilde{\alpha}+1}\left(k x_{0}\right)\right) .
$$

We have now to impose the subsidiary condition (5), which in terms of the Lorentz index fields reads

$$
x_{0} \partial_{\mu} \tilde{A}_{\mu}-d \tilde{A}_{0}=0
$$

Inserting (7) and (11) into (12) yields

$$
a_{0}\left(\tilde{\alpha}-\frac{d}{2}+1\right)=i a_{i} k_{i}
$$

which determines $a_{0}$ in the generic case of massive vector fields, but leaves it undetermined in the massless case. In order to find a prescription, which is valid for both cases, let us first impose the boundary conditions on the fields $\tilde{A}_{i}$. It is useful to write

$$
a_{i}=b_{i}+b k_{i}
$$

Setting $x_{0}=\epsilon$ in (11) we then find

$$
b_{i} K_{\tilde{\alpha}}+k_{i}\left[b K_{\tilde{\alpha}}+i a_{0} \frac{\epsilon}{k} K_{\tilde{\alpha}+1}\right]=\epsilon^{-\frac{d}{2}} \tilde{A}_{\epsilon, i}(\mathbf{k}),
$$

where the argument $k \epsilon$ of the modified Bessel functions has been omitted and $\tilde{A}_{\epsilon, i}(\mathbf{k})$ denotes the Fourier transform of the Dirichlet boundary value of the field $\tilde{A}_{i}$. We can determine $b_{i}$ and $a_{0}$ from (15) by identifying the first term on the l.h.s. with the r.h.s. and demanding that the second term on the l.h.s. is zero. This yields

$$
\begin{aligned}
b_{i} & =\epsilon^{-\frac{d}{2}} \frac{\tilde{A}_{\epsilon, i}(\mathbf{k})}{K_{\tilde{\alpha}}}, \\
a_{0} & =i \frac{k b K_{\tilde{\alpha}}}{\epsilon K_{\tilde{\alpha}+1}} .
\end{aligned}
$$

Substituting (14) and (17) into (13) we find the missing coefficcient

$$
b=\frac{b_{i} k_{i}}{k^{2}} \frac{k \epsilon K_{\tilde{\alpha}+1}}{(1-\tilde{\Delta}) K_{\tilde{\alpha}}-k \epsilon K_{\tilde{\alpha}-1}},
$$

where a functional relation of the modified Bessel functions has been used to rearrange the denominator and we have defined $\tilde{\Delta}=\tilde{\alpha}+d / 2$. 
Let us use the AdS/CFT correspondence to calculate the two-point functions of currents $J_{i}$, which couple to the massive vector fields $A_{0, i}$. After integration by parts and using (四) the action (3) takes the value

$$
I=-\frac{1}{2} \int d^{d} x \epsilon^{-d} \tilde{A}_{\epsilon, i}\left[-\tilde{A}_{\epsilon, i}+\epsilon \tilde{F}_{\epsilon, 0 i}\right]
$$

where $\tilde{F}_{0 i}=\partial_{0} \tilde{A}_{i}-\partial_{i} \tilde{A}_{0}$ contains the interesting part. Using the solutions ([0) and (11) with the coefficcients obtained in (14), (16), (17) and (18) one finds

$$
\begin{aligned}
\tilde{F}_{\epsilon, 0 i}=\left(\frac{d}{2}-\tilde{\alpha}\right) & \frac{1}{\epsilon} \tilde{A}_{\epsilon, i} \\
& +\int \frac{d^{d} k}{(2 \pi)^{d}} \mathrm{e}^{-i \mathbf{k} \cdot \mathbf{x}} \tilde{A}_{\epsilon, j}(\mathbf{k}) k \frac{K_{\tilde{\alpha}-1}}{K_{\tilde{\alpha}}}\left[-\delta_{i j}+\frac{k_{i} k_{j}}{k^{2}} \frac{k \epsilon K_{\tilde{\alpha}+1}}{(\tilde{\Delta}-1) K_{\tilde{\alpha}}+k \epsilon K_{\tilde{\alpha}-1}}\right] .
\end{aligned}
$$

We take the limit $\epsilon \rightarrow 0$ by substituting the first terms of the series expansion of the modified Bessel functions in (20). The series expansion is given by

$$
K_{\nu}(z)=z^{-\nu} 2^{\nu-1} \Gamma(\nu)\left[1-\left(\frac{z}{2}\right)^{2 \nu} \frac{\Gamma(1-\nu)}{\Gamma(1+\nu)}+\cdots\right],
$$

where the dots indicate terms of order $z^{2 n}$ and $z^{2 \nu+2 n}(n=1,2, \ldots)$. Our experience from the scalar field [16] tells us that the relevant terms are proportional to $k^{2 \tilde{\alpha}} \delta_{i j}$ and $k^{2 \tilde{\alpha}-2} k_{i} k_{j}$. We obtain these by keeping only the leading order terms for the denominators in (20) and using the appropriate terms for the numerators. In particular, the term $k^{2 \tilde{\alpha}}$ from (21) is needed only for $K_{\tilde{\alpha}-1}$ in the numerator of $(20)$. One obtains

$$
\begin{aligned}
\tilde{F}_{\epsilon, 0 i}=\left(\frac{d}{2}-\tilde{\alpha}\right) \frac{1}{\epsilon} \tilde{A}_{\epsilon, i} & +\left(\frac{\epsilon}{2}\right)^{2 \tilde{\alpha}-1} \frac{\Gamma(1-\tilde{\alpha})}{\Gamma(\tilde{\alpha})} \\
& \times \int \frac{d^{d} k}{(2 \pi)^{d}} \mathrm{e}^{-i \mathbf{k} \cdot \mathbf{x}} \tilde{A}_{\epsilon, j}(\mathbf{k})\left(-k^{2 \tilde{\alpha}} \delta_{i j}+\frac{2 \tilde{\alpha}}{\tilde{\Delta}-1} k^{2 \tilde{\alpha}-2} k_{i} k_{j}+\cdots\right),
\end{aligned}
$$

where the dots denote all other terms representing either contact terms in the two-point function or terms of higer order in $\epsilon$. Performing the integrals in (22) and inserting the result into (19) yields

$$
\begin{aligned}
I=\frac{1}{2} & \left(\tilde{\alpha}+1-\frac{d}{2}\right) \int d^{d} x \epsilon^{-d} \tilde{A}_{\epsilon, i}(\mathbf{x}) \tilde{A}_{\epsilon, i}(\mathbf{x}) \\
& -\frac{1}{2} \frac{2 \tilde{c} \tilde{\alpha} \tilde{\Delta}}{\tilde{\Delta}-1} \int d^{d} x d^{d} y \tilde{A}_{\epsilon, i}(\mathbf{x}) \tilde{A}_{\epsilon, i}(\mathbf{y}) \frac{\epsilon^{2(\tilde{\Delta}-d)}}{|\mathbf{x}-\mathbf{y}|^{2 \tilde{\Delta}}}\left(\delta_{i j}-2 \frac{(x-y)_{i}(x-y)_{j}}{|\mathbf{x}-\mathbf{y}|^{2}}\right)+\cdots,
\end{aligned}
$$

with

$$
\tilde{c}=\frac{\Gamma(\tilde{\Delta})}{\pi^{\frac{d}{2}} \Gamma(\tilde{\alpha})}
$$


Identifying

$$
A_{0, i}(\mathbf{x})=\lim _{\epsilon \rightarrow 0} \epsilon^{\tilde{\Delta}-d} \tilde{A}_{\epsilon, i}(\mathbf{x})
$$

and using the AdS/CFT correspondence of the form

$$
\exp \left(-I_{A d S}\right) \equiv\left\langle\exp \left(\int d^{d} x J_{j}(\mathbf{x}) A_{0, j}(\mathbf{x})\right)\right\rangle
$$

we can read off from (23) the finite distance two-point function as

$$
\left\langle J_{i}(\mathbf{x}) J_{j}(\mathbf{y})\right\rangle=\frac{2 \tilde{c} \tilde{\alpha} \tilde{\Delta}}{\tilde{\Delta}-1}\left(\delta_{i j}-2 \frac{(x-y)_{i}(x-y)_{j}}{|\mathbf{x}-\mathbf{y}|^{2}}\right)|\mathbf{x}-\mathbf{y}|^{-2 \tilde{\Delta}},
$$

which is of the form dictated by conformal invariance. It shows in particular that $J_{i}$ has the conformal dimension $\tilde{\Delta}$. This is of course as expected, but in view of the fact that the integrals in (22) have to combine to give exactly the terms in parenthesis in (26) it is a non-trivial check of our derivation. Moreover, our result coincides for the massless case with the one obtained in [17].

In contrast to the two-point function, which is determined by a boundary integral, interactions involve integrals over the volume of $\mathrm{AdS}_{d+1}$. Hence, higher correlation functions are not sensitive to when the limit $\epsilon \rightarrow 0$ is taken and we shall take it for the fields $A_{\mu}$.

Substituting (14), (16), (17) and (18) into (11) and replacing $K_{\nu}(k \epsilon)$ by the leading order term of its asymptotic expansion (21) one finds

$$
A_{i}^{b u l k}(x)=\frac{\tilde{c} \tilde{\Delta}}{\tilde{\Delta}-1} \int d^{d} y A_{0, j}(\mathbf{y}) \frac{x_{0}^{\tilde{\Delta}-1}}{\left(x_{0}^{2}+|\mathbf{x}-\mathbf{y}|^{2}\right)^{\tilde{\Delta}}}\left(\delta_{i j}-2 \frac{(x-y)_{i}(x-y)_{j}}{x_{0}^{2}+|\mathbf{x}-\mathbf{y}|^{2}}\right) .
$$

Similarly, taking the limit in (7) yields

$$
A_{0}^{b u l k}(x)=-\frac{2 \tilde{c} \tilde{\Delta}}{\tilde{\Delta}-1} \int d^{d} y A_{0, j}(\mathbf{y}) \frac{x_{0}^{\tilde{\Delta}}(x-y)_{j}}{\left(x_{0}^{2}+|\mathbf{x}-\mathbf{y}|^{2}\right)^{\tilde{\Delta}+1}}
$$

\section{The Free Dirac Field}

Let us start with the action

$$
I[\bar{\psi}, \psi]=\int d^{d+1} x \sqrt{g} \bar{\psi}(x)(\not D-m) \psi(x)+G \int d^{d} x \sqrt{h} \bar{\psi}(\mathbf{x}) \psi(\mathbf{x}),
$$

where we supplemented the dynamical bulk action with a surface term [18] with an as yet undetermined coefficcient $G$. The surface term is necessary in order to obtain a two-point function of spinors in the boundary conformal field theory. The equation of motion for $\psi$ derived from the action (29) is the Dirac equation

$$
(\not D-m) \psi(x)=\left(x_{0} \gamma_{\mu} \partial_{\mu}-\frac{d}{2} \gamma_{0}-m\right) \psi(x)=0
$$


where the matrices $\gamma_{\mu}$ are the Dirac matrices of $d+1$ dimensional Euclidian space, i.e. $\gamma_{\mu} \gamma_{\nu}+\gamma_{\nu} \gamma_{\mu}=2 \delta_{\mu \nu}$. Acting with $\gamma_{\mu} \partial_{\mu}$ on (30) one obtains the second order differential equation

$$
\left[\partial_{\mu} \partial_{\mu}-\frac{d}{x_{0}} \partial_{0}-\frac{1}{x_{0}^{2}}\left(m^{2}-\frac{d^{2}}{4}-\frac{d}{2}-\gamma_{0} m\right)\right] \psi(x)=0 .
$$

The solution of (31), which does not diverge for $x_{0} \rightarrow \infty$, is obtained in a similar fashion as in the scalar and vector cases and is given by

$$
\psi(x)=\int \frac{d^{d} k}{(2 \pi)^{d}} \mathrm{e}^{-i \mathbf{k} \cdot \mathbf{x}} x_{0}^{\frac{d+1}{2}}\left(a^{+}(\mathbf{k}) K_{m-\frac{1}{2}}\left(k x_{0}\right)+a^{-}(\mathbf{k}) K_{m+\frac{1}{2}}\left(k x_{0}\right)\right)
$$

where the spinors $a^{ \pm}$satisfy $\gamma_{0} a^{ \pm}= \pm a^{ \pm}$. The expression (32) is in general not a solution of the Dirac equation (30). In fact, substituting (32) into (30) we find that the spinors $a^{+}$ and $a^{-}$must be related by

$$
a^{-}=\frac{i}{k} k_{i} \gamma_{i} a^{+}
$$

Our next task is to impose boundary conditions on the solution (32). However, there is a major difference to the scalar and vector cases. The origin of this difference lies in the nature of the differential equations, which serve as the equations of motion for the fields. In the scalar case [16 and vector case (cf. Sec. 2) we have second order differential equations. Hence, we could impose two sets of boundary data, namely the field and its derivative. Instead of the latter we demand that the field be well behaved in the volume of $A d S_{d+1}$, i.e. for $x_{0} \rightarrow \infty$, which yields a unique solution to the Dirichlet problem. On the other hand, the Dirac equation (30) is a first order differential equation. The $x_{0} \rightarrow \infty$ behaviour of the solutions of the Dirac equation is crucial from the AdS field theory point of view and cannot be abandoned. Hence, only half of the general solutions are available for fitting the boundary data, which means that only half the components of the spinor $\psi$ can be prescribed on the boundary, the other half being fixed by a relation, which will be determined in a moment. This result is important also from a CFT point of view. Considering the boundary term of the action (29) we realize that, if one could prescribe the entire boundary spinor, then there would be only a contact term in the CFT two-point function. The trade-off is that we can obtain only correlators for spinors, which have half the number of components as the field $\psi$. This means that the boundary spinors are Weyl or Dirac spinors for $d$ even or odd, respectively [18].

Letting $x_{0}=\epsilon$ in (32) we find

$$
\psi_{\epsilon}(\mathbf{k})=\epsilon^{\frac{d+1}{2}}\left(K_{m-\frac{1}{2}}+i \frac{k_{i} \gamma_{i}}{k} K_{m+\frac{1}{2}}\right) a^{+}(\mathbf{k}),
$$


where $\psi_{\epsilon}(\mathbf{k})$ is the Fourier transform of the boundary spinor and we have omitted the argument $k \epsilon$ of the modified Bessel functions. We can determine $a^{+}$from (34) in two ways, namely by

$$
a^{+}(\mathbf{k})=\epsilon^{-\frac{d+1}{2}} \frac{\psi_{\epsilon}^{+}(\mathbf{k})}{K_{m-\frac{1}{2}}}
$$

or

$$
a^{+}(\mathbf{k})=\epsilon^{-\frac{d+1}{2}} \frac{k_{i} \gamma_{i}}{i k} \frac{\psi_{\epsilon}^{-}(\mathbf{k})}{K_{m+\frac{1}{2}}}
$$

where $\psi_{\epsilon}^{ \pm}=\frac{1}{2}\left(1 \pm \gamma_{0}\right) \psi_{\epsilon}$. Substituting (36) into (35) we find that $\psi_{\epsilon}^{+}$and $\psi_{\epsilon}^{-}$are related by

$$
\psi_{\epsilon}^{+}(\mathbf{k})=-i \frac{k_{i} \gamma_{i}}{k} \frac{K_{m-\frac{1}{2}}}{K_{m+\frac{1}{2}}} \psi_{\epsilon}^{-}(\mathbf{k}) .
$$

The question as to which of the functions $\psi_{\epsilon}^{ \pm}$should be used as boundary data is, in general, not a matter of choice, but is dictated by the $\epsilon \rightarrow 0$ limit. Here we have to distinguish three cases. If $m>0, K_{m-\frac{1}{2}}$ diverges slower than $K_{m+\frac{1}{2}}$ for $\epsilon \rightarrow 0$ and thus we find that $\psi_{\epsilon}^{+} \rightarrow 0$, if we fix $\psi_{\epsilon}^{-}$. This is in agreement with the condition found in [18]. On the other hand, we cannot prescribe $\psi_{\epsilon}^{+}$for $m>0$, as $\psi_{\epsilon}^{-}$would then diverge. The case $m<0$ is just vice versa. For $m=0$ we have $K_{-\frac{1}{2}}=K_{\frac{1}{2}}$ and hence one can prescribe either of the functions $\psi_{\epsilon}^{ \pm}$.

We shall in the following consider the case $m \geq 0$. Inserting (36) and (33) into (32) we finally find

$$
\psi(x)=\int \frac{d^{d} k}{(2 \pi)^{d}} \mathrm{e}^{-i \mathbf{k} \cdot \mathbf{x}}\left(\frac{x_{0}}{\epsilon}\right)^{\frac{d+1}{2}}\left(-i \frac{k_{i} \gamma_{i}}{k} K_{m-\frac{1}{2}}\left(k x_{0}\right)+K_{m+\frac{1}{2}}\left(k x_{0}\right)\right) \frac{\psi_{\epsilon}^{-}(\mathbf{k})}{K_{m+\frac{1}{2}}(k \epsilon)} .
$$

In a similar fashion one can solve the equation of motion for the conjugate spinor,

$$
\bar{\psi}(x)(\overleftarrow{D D}+m)=\bar{\psi}(x)\left(\overleftarrow{\partial}_{\mu} \gamma_{\mu} x_{0}-\frac{d}{2} \gamma_{0}+m\right)=0
$$

The solution in the case $m \geq 0$ is

$$
\bar{\psi}(x)=\int \frac{d^{d} k}{(2 \pi)^{d}} \mathrm{e}^{-i \mathbf{k} \cdot \mathbf{x}}\left(\frac{x_{0}}{\epsilon}\right)^{\frac{d+1}{2}} \frac{\bar{\psi}_{\epsilon}^{+}(\mathbf{k})}{K_{m+\frac{1}{2}}(k \epsilon)}\left(i \frac{k_{i} \gamma_{i}}{k} K_{m-\frac{1}{2}}\left(k x_{0}\right)+K_{m+\frac{1}{2}}\left(k x_{0}\right)\right),
$$

where $\bar{\psi}_{\epsilon}^{ \pm}=\bar{\psi}_{\epsilon} \frac{1}{2}\left(1 \pm \gamma_{0}\right)$. Again we find a relation between the components of the boundary spinor, which is given by

$$
\bar{\psi}_{\epsilon}^{-}(\mathbf{k})=\bar{\psi}_{\epsilon}^{+}(\mathbf{k}) i \frac{k_{i} \gamma_{i}}{k} \frac{K_{m-\frac{1}{2}}}{K_{m+\frac{1}{2}}}
$$


Let us turn now to the two-point function for the boundary spinors $\chi^{+}$and $\bar{\chi}^{-}$, which couple to $\bar{\psi}_{0}^{+}$and $\psi_{0}^{-}$, respectively. Inserting the solutions of the equations of motion into the action (29), the bulk term vanishes and the surface term can be written as

$$
I=G \epsilon^{-d} \int \frac{d^{d} k}{(2 \pi)^{d}}\left(\bar{\psi}^{+}(\mathbf{k}) \psi^{+}(-\mathbf{k})+\bar{\psi}^{-}(\mathbf{k}) \psi^{-}(-\mathbf{k})\right)
$$

Using the relations (37) and (41) one finds

$$
I=G \epsilon^{-d} \int d^{d} x d^{d} y \int \frac{d^{d} k}{(2 \pi)^{d}} \mathrm{e}^{i \mathbf{k} \cdot(\mathbf{x}-\mathbf{y})} \bar{\psi}_{\epsilon}^{+}(\mathbf{x})\left(2 i \frac{k_{i} \gamma_{i}}{k} \frac{K_{m-\frac{1}{2}}}{K_{m+\frac{1}{2}}}\right) \psi_{\epsilon}^{-}(\mathbf{y}) .
$$

We use the expansion (21) for the modified Bessel functions in the numerator and the leading order term in the denominator. Hence, we find after integration

$$
I=-2 \hat{c} G \int d^{d} x d^{d} y \bar{\psi}_{0}^{+}(\mathbf{x}) \frac{\gamma_{i}\left(x_{i}-y_{i}\right)}{|\mathbf{x}-\mathbf{y}|^{d+2 m+1}} \psi_{0}^{-}(\mathbf{y}),
$$

where we defined

$$
\psi_{0}^{-}=\lim _{\epsilon \rightarrow 0} \epsilon^{m-\frac{d}{2}} \psi_{\epsilon}^{-} \quad \text { and } \quad \bar{\psi}_{0}^{+}=\lim _{\epsilon \rightarrow 0} \epsilon^{m-\frac{d}{2}} \bar{\psi}_{\epsilon}^{+}
$$

for the $\epsilon \rightarrow 0$ limit and

$$
\hat{c}=\frac{\Gamma\left(\frac{d+1}{2}+m\right)}{\pi^{\frac{d}{2}} \Gamma\left(m+\frac{1}{2}\right)} .
$$

In the case $m=0$ the $k$ integral in (43) can be done without the asymptotic expansion and leads to the same result. Using the AdS/CFT correspondence

$$
\exp \left(-I_{A d S}\right) \equiv\left\langle\exp \left(\int d^{d} x\left(\bar{\chi}^{-} \psi_{0}^{-}+\bar{\psi}_{0}^{+} \chi^{+}\right)\right)\right\rangle
$$

the two-point function reads

$$
\left\langle\chi^{+}(\mathbf{x}) \bar{\chi}^{-}(\mathbf{y})\right\rangle=2 \hat{c} G \frac{\gamma_{i}\left(x_{i}-y_{i}\right)}{|\mathbf{x}-\mathbf{y}|^{d+2 m+1}}
$$

Hence, the spinors $\chi$ and $\bar{\chi}$ have the conformal dimension $m+\frac{d}{2}$. Our result agrees up to the appropriate normalization with the one found in 18 .

For calculating interactions we are interested in the bulk behaviour of the spinors $\psi$ and $\bar{\psi}$. It is obtained by replacing $K_{m+\frac{1}{2}}(k \epsilon)$ by the leading order term of its asymptotic expansion in (38) and (40). One finds the expressions

$$
\psi^{b u l k}(x)=\hat{c} \int d^{d} y\left[x_{0}-\gamma_{i}\left(x_{i}-y_{i}\right)\right]\left(x_{0}^{2}+|\mathbf{x}-\mathbf{y}|^{2}\right)^{-\frac{d+1}{2}-m} x_{0}^{\frac{d}{2}+m} \psi_{0}^{-}(\mathbf{y})
$$


and

$$
\bar{\psi}^{\text {bulk }}(x)=\hat{c} \int d^{d} y \bar{\psi}_{0}^{+}(\mathbf{y})\left[x_{0}+\gamma_{i}\left(x_{i}-y_{i}\right)\right]\left(x_{0}^{2}+|\mathbf{x}-\mathbf{y}|^{2}\right)^{-\frac{d+1}{2}-m} x_{0}^{\frac{d}{2}+m},
$$

which coincide with those in [18 up to normalization. A good check of the derivation of

these expressions is provided by the case $m=0$. Since $K_{ \pm \frac{1}{2}}(z)=\sqrt{\frac{\pi}{2 z}} \mathrm{e}^{-z}$, it is possible to carry out the integral in (38) with the result

$$
\psi(x)=\int d^{d} y \frac{\Gamma\left(\frac{d+1}{2}\right)}{\pi^{\frac{d}{2}} \Gamma\left(\frac{1}{2}\right)} x_{0}^{\frac{d}{2}}\left[\left(x_{0}-\epsilon\right)^{2}+|\mathbf{x}-\mathbf{y}|^{2}\right]^{-\frac{d+1}{2}}\left[x_{0}-\epsilon-\gamma_{i}\left(x_{i}-y_{i}\right)\right] \psi_{0}^{-}(\mathbf{y}) .
$$

\section{Interaction between Spinor and Gauge Fields}

Calculating the first order interaction between the spinor and massless vector fields serves two purposes. First, it provides another detail of the AdS/CFT correspondence in form of the vector-spinor-spinor three-point function. In contrast to the scalar three-point function, conformal symmetry does not fix, but only restricts the form of this particular three-point function [1]. Hence, the calculation will yield more than just a coefficcient in front of a universal function. Second, a check of the Ward identity corresponding to the gauge invariance will reveal that no supplementary surface term of the order of the gauge coupling is needed.

We shall use the action for minimally coupled spinor and gauge fields, together with the spinor surface term,

$$
I=\int d^{d+1} x \sqrt{g}\left[\frac{1}{4} F_{\mu \nu} F^{\mu \nu}+\bar{\psi}(\not D-i q A-m) \psi\right]+G \int d^{d} x \sqrt{h} \bar{\psi} \psi
$$

The equations of motion derived from (51) are

$$
\begin{aligned}
\nabla_{\mu} F^{\mu \nu} & =-i q \mathrm{e}_{a}^{\nu} \bar{\psi} \gamma_{a} \psi, \\
(\not D-m) \psi & =i q A \psi
\end{aligned}
$$

and its conjugate

$$
\bar{\psi}(\overleftarrow{D}+m)=-i q \bar{\psi} A
$$

We split the gauge field into its free part $A^{(0)}$ and the remainder $A^{(1)}$. Substituting (53) into (51) and using the equation of motion for $F^{(0)}$, we find

$$
I=\int d^{d} x \epsilon^{-d}\left(-\frac{1}{2 \epsilon} A_{i}^{(0)} F^{(0), 0 i}-\frac{1}{\epsilon} A_{i}^{(1)} F^{(0), 0 i}+G \bar{\psi} \psi\right)+\mathcal{O}\left(q^{2}\right) .
$$

Most importantly, the bulk terms vanish! Moreover, using the appropriate Green's function to calculate $A^{(1)}$ (cf. [16] for the scalar field analogue), we realize that also the second term 
in (55) is zero. The first term only yields the two-point function for the conserved currents $J$. However, the last term will give the two-point function for the spinors and the three-point function coupling $J$ and the spinors. This surprising fact comes about as follows. Going back to the derivation of the spinor two-point function, we realize that it was generated by the relations (37) and (41) between the + and - components of the spinors on the boundary. These relations will be altered by the presence of the interaction. Writing

$$
\begin{aligned}
\psi(x) & =\psi^{(0)}(x)+\psi^{(1)}(x)+\mathcal{O}\left(q^{2}\right), \\
\psi^{(1)}(x) & =i q \int d^{d+1} y \sqrt{g} S(x, y) A(y) \psi^{(0)}(y),
\end{aligned}
$$

where $S(x, y)$ is the spinor Green's function defined by

$$
\left(\not D_{x}-m\right) S(x, y)=\frac{\delta(x-y)}{\sqrt{g(x)}}
$$

we find using (37)

$$
\psi^{+}(\mathbf{k})=-i \frac{k_{i} \gamma_{i}}{k} \frac{K_{m-\frac{1}{2}}}{K_{m+\frac{1}{2}}} \psi^{-}(\mathbf{k})+\frac{1+\gamma_{0}}{2}\left(1+i \frac{k_{i} \gamma_{i}}{k} \frac{K_{m-\frac{1}{2}}}{K_{m+\frac{1}{2}}}\right) \psi^{(1)}(\mathbf{k})+\mathcal{O}\left(q^{2}\right),
$$

where we omitted the argument $k \epsilon$ of the modified Bessel functions. Similarly, one finds for the conjugate field

$$
\begin{aligned}
\bar{\psi}(x) & =\bar{\psi}^{(0)}(x)+\bar{\psi}^{(1)}(x)+\mathcal{O}\left(q^{2}\right), \\
\bar{\psi}^{(1)}(x) & =i q \int d^{d+1} y \sqrt{g} \bar{\psi}^{(0)}(y) A(y) \bar{S}(y, x),
\end{aligned}
$$

with"

$$
\bar{S}(y, x)(\overleftarrow{D D}+m)=-\frac{\delta(x-y)}{\sqrt{g(x)}}
$$

and, using (41)

$$
\bar{\psi}^{-}(\mathbf{k})=\bar{\psi}^{+}(\mathbf{k}) i \frac{k_{i} \gamma_{i}}{k} \frac{K_{m-\frac{1}{2}}}{K_{m+\frac{1}{2}}}+\bar{\psi}^{(1)}(\mathbf{k})\left(1-i \frac{k_{i} \gamma_{i}}{k} \frac{K_{m-\frac{1}{2}}}{K_{m+\frac{1}{2}}}\right) \frac{1-\gamma_{0}}{2}+\mathcal{O}\left(q^{2}\right) .
$$

Substituting (59) and (63) into the spinor surface term in the form (42), one finds that the contribution to the action of first order in $q$ is

$$
\begin{aligned}
I^{(1)}=G \epsilon^{-d} \int \frac{d^{d} k}{(2 \pi)^{d}}\left[\left(\bar{\psi}^{(0)+}(\mathbf{k})-\bar{\psi}^{(0)-}(\mathbf{k})\right) \psi^{(1)}(-\mathbf{k})\right. & \\
& \left.-\bar{\psi}^{(1)}(-\mathbf{k})\left(\psi^{(0)+}(\mathbf{k})-\psi^{(0)-}(\mathbf{k})\right)\right]+\mathcal{O}\left(q^{2}\right) .
\end{aligned}
$$

\footnotetext{
${ }^{1}$ The relation between $\bar{S}$ and $S$ is of no importance here.
} 
On the other hand, from (52) and (58) one can obtain

$$
\psi^{(0)}(x)=\epsilon^{-d} \int d^{d} y \bar{S}(x, \mathbf{y})\left(\psi^{(0)+}(\mathbf{y})-\psi^{(0)-}(\mathbf{y})\right)
$$

and

$$
\bar{\psi}^{(0)}(x)=-\epsilon^{-d} \int d^{d} y\left(\bar{\psi}^{(0)+}(\mathbf{y})-\bar{\psi}^{(0)-}(\mathbf{y})\right) S(\mathbf{y}, x),
$$

respectively. Inserting (57), (66), (61) and (65) into (64) one then finds

$$
I^{(1)}=-2 G i q \int d^{d+1} x \sqrt{g} \bar{\psi}^{(0)} A \psi^{(0)} .
$$

Eqn. (67) has the same form as the minimal coupling term, but is multiplied by $2 G$. It is determined by a bulk integral, which means that the bulk behaviour for the fields can be used. Substituting (27), (28) (with $\tilde{\Delta}=d-1$ ), (48) and (49) into (67), the following tedious calculation involves Feynman parameterization of the denominator and heavy numerator algebra. The result is

$$
\begin{aligned}
\left\langle J_{j}\left(\mathbf{x}_{2}\right) \chi^{+}\left(\mathbf{x}_{1}\right) \bar{\chi}^{-}\left(\mathbf{x}_{3}\right)\right\rangle & =\frac{-i G q \hat{c} \Gamma\left(\frac{d}{2}\right)}{\pi^{\frac{d}{2}}(d-1+2 m)} \\
\times & {\left[(d-2) \gamma_{i} \gamma_{j} \gamma_{k} \frac{x_{12 i} x_{23 k}}{x_{12}^{d} x_{23}^{d} x_{13}^{2 m+1}}+(2 m+1) \gamma_{i} x_{13 i} \frac{x_{12}^{2} x_{23 j}+x_{23}^{2} x_{12 j}}{x_{12}^{d} x_{23}^{d} x_{13}^{2 m+3}}\right], }
\end{aligned}
$$

where $\mathbf{x}_{a b}=\mathbf{x}_{a}-\mathbf{x}_{b}$. After further algebra one finds that (68) can be written in the form

$$
\begin{aligned}
&\left\langle J_{j}\left(\mathbf{x}_{2}\right) \chi^{+}\left(\mathbf{x}_{1}\right) \bar{\chi}^{-}\left(\mathbf{x}_{3}\right)\right\rangle=\frac{-i G q \hat{c} \Gamma\left(\frac{d}{2}\right)}{\pi^{\frac{d}{2}}(d-1+2 m)} \frac{1}{x_{12}^{d} x_{23}^{d-2} x_{13}^{2 m}} \frac{\gamma_{i} x_{13 i}}{x_{13}} \\
& \times\left(\delta_{j k}-2 \frac{x_{23 j} x_{23 k}}{x_{23}^{2}}\right)\left(\frac{x_{13 l}}{x_{13}^{2}}-\frac{x_{23 l}}{x_{23}^{2}}\right)\left[(d-2) \gamma_{l} \gamma_{k}+(2 m+1) \delta_{k l}\right],
\end{aligned}
$$

which is a specific case of the general expression dictated by conformal invariance [1].

Finally, let us confirm the Ward identity [22]

$$
\frac{\partial}{\partial x_{2}^{j}}\left\langle J_{j}\left(\mathbf{x}_{2}\right) \chi^{+}\left(\mathbf{x}_{1}\right) \bar{\chi}^{-}\left(\mathbf{x}_{3}\right)\right\rangle=-i q\left\langle\chi^{+}\left(\mathbf{x}_{1}\right) \bar{\chi}^{-}\left(\mathbf{x}_{3}\right)\right\rangle\left[\delta\left(\mathbf{x}_{23}\right)-\delta\left(\mathbf{x}_{12}\right)\right] .
$$

From (68) one finds

$$
\frac{\partial}{\partial x_{2}^{j}}\left\langle J_{j}\left(\mathbf{x}_{2}\right) \chi^{+}\left(\mathbf{x}_{1}\right) \bar{\chi}^{-}\left(\mathbf{x}_{3}\right)\right\rangle=-i G q 2 \hat{c} \frac{\gamma_{i} x_{13 i}}{x_{13}^{d+2 m+1}}\left[\delta\left(\mathbf{x}_{23}\right)-\delta\left(\mathbf{x}_{12}\right)\right] .
$$

Comparing (71) and (47) with (70) we see that the Ward identity is satisfied. This result is significant, since is tells us that, to first order in $q$, no supplementary surface term except the one used already for the free Dirac field is required in the action for interacting fields. 


\section{Conclusions}

In the present paper we used the AdS/CFT correspondence to calculate CFT correlators from the classical AdS theories of vector and Dirac fields. We took care to address the proper treatment of the $\epsilon \rightarrow 0$ limit when calculating the two-point functions. As for the scalar field [16, 17], this was particularly important for the vector field with non zero mass.

Our calculation for the free Dirac field revealed the full details as to why only half the number of spinor components can be given as boundary data. For odd $d$ this is exactly what one wants, because the boundary spinor representation has only half the number of components as the bulk spinors. For even $d$ the dimensions of the spinor representations are the same and $\gamma_{0}$ acts as the chirality operator on the boundary spinors. This means that for even $d$ we calculated only the correlation functions for chiral spinors. However, the formalism can be extended to Dirac spinors by coupling $\chi^{-}$to an AdS spinor $\psi_{1}$ with positive mass $m$ and $\chi^{+}$to a field $\psi_{2}$ with mass $-m$.

Minimally coupling the Dirac and massless vector field, we calculated the CFT vectorspinor-spinor three-point function. The result should be interesting from a CFT point of view, as the form of this correlator is not totally fixed by conformal invariance. Thus, our result could indicate, which CFT is obtained by the AdS/CFT correspondence. Finally, we confirmed the validity of the Ward identity and found that no interaction surface terms are required in the action.

\section{Acknowledgements}

We would like to thank D. Freedman and K. Sfetsos for interesting correspondence.

This work was supported in part by an operating grant from NSERC. W. M. gratefully acknowledges the support with a Graduate Fellowship from Simon Fraser University.

\section{References}

[1] H. Osborn and A. Petkou, Ann. Phys. 231, 311 (1994)

[2] J. Erdmenger and H. Osborn, Nucl. Phys. B 483, 431 (1997)

[3] J. Maldacena, The large $N$ limit of superconformal field theories and supergravity. hepth/9711200

[4] S. Gubser, I. R. Klebanov and A. M. Polyakov, Gauge theory correlators from noncritical string theory. hep-th/9802109

[5] E. Witten, Anti de Sitter space and holography. hep-th/9802150 
[6] C. Frønsdal, Rev. Mod. Phys. 37, 221 (1965)

[7] C. Frønsdal, Phys. Rev. D 10, 589 (1974)

[8] C. Frønsdal, Phys. Rev. D 12, 3810 (1975)

[9] C. Frønsdal, Phys. Rev. D 12, 3819 (1975)

[10] C. P. Burgess and C. A. Lütken, Phys. Lett. 153B, 137 (1985)

[11] T. Inami and H. Ooguri, Prog. Theor. Phys. 73, 1051 (1985)

[12] B. Allen and T. Jacobson Comm. Math. Phys. 103, 669 (1986)

[13] B. Allen and C. A. Lütken, Comm. Math. Phys. 106, 201 (1986)

[14] C. J. C. Burges, D. Z. Freedman, S. Davis and G. W. Gibbons, Annals of Physics 167, 285 (1986)

[15] I. Y. Aref'eva and I. V. Volovich, On large $N$ conformal theories, field theories in anti-de Sitter space and singletons. hep-th/9803028

[16] W. Mück and K. S. Viswanathan, Conformal field theory correlators from classical scalar field theory on anti-de Sitter space. hep-th/9804035

[17] D. Z. Freedman, S. D. Mathur, A. Matusis and L. Rastelli, Correlation functions in the $C F T_{d} / A d S_{d+1}$ correspondence. hep-th/9804058

[18] M. Henningson and K. Sfetsos, Spinors and the AdS/CFT correspondence. hepth/9803251

[19] H. Liu and A. A. Tseytlin, D=4 Super Yang-Mills, D=5 gauge supergravities and $D=4$ conformal supergravity. hep-th/9804083

[20] T. Banks and M. B. Green, Non-perturbative effects in $A d S_{5} \times S^{5}$ string theory and $d=4$ SUSY Yang Mills. hep-th/9804170

[21] G. Chalmers, H. Nastase, K. Schalm and R. Siebelink, R-current correlators in $N=4$ Super Yang-Mills theory from anti-de Sitter supergravity. hep-th/9805105

[22] P. Di Francesco, P. Mathieu and D. Sénéchal, Conformal Field Theory. Springer Verlag (1997) 